studies, the performances of endobronchial elastography will have to be assessed against the results of mediastinoscopy as the gold standard approach.

From this experience, we conclude that transbronchial biomechanical analysis can be performed during bronchial endoscopic ultrasound. Tracheobronchial cartilage does not appear to interfere with collection of this type of information. The preliminary results suggest that elastography could possibly improve the diagnostic yield of bronchial endoscopic ultrasound, as has already been demonstrated in gastrointestinal endoscopy. This is all the more important as endobronchial ultrasonography has a low negative predictive value regarding the neoplastic involvement of mediastinal lymph nodes (see [11] for an example). We propose that our preliminary results justify the setting-up of large-scale studies to precisely describe the operating characteristics of bronchial endoscopic ultrasound elastography. It would also be interesting to determine whether elastography could improve guidance of TBNA in heterogeneous lymph node masses or limit the number of TBNAs performed, by primarily targeting the lymph nodes most likely to be malignant.

\section{Valery Trosini-Désert*,\#, Florence Jeny*, Laurent Taillade Stephane Vignot ${ }^{\oplus}$, Hedi Zribi*, Frédérique Capron ${ }^{+}$and Thomas Similowski* \\ *Assistance Publique - Hôpitaux de Paris, Groupe Hospitalier Pitié-Salpêtrière Charles Foix, Dept of Respiratory and Critical Care Medicine, "Assistance Publique - Hôpitaux de Paris, Groupe Hospitalier Pitié-Salpêtrière Charles Foix, Dept of Medical Oncology, ${ }^{+}$Assistance Publique - Hôpitaux de Paris, Groupe Hospitalier Pitié-Salpêtrière Charles Foix, Dept of Pathology, and "Groupe d'Endoscopie de Langue Française, Société de Pneumologie de Langue Française, Paris, France.}

Correspondence: V. Trosini-Désert, Service de Pneumologie et de Réanimation Médicale, Groupe Hospitalier Pitié-Salpêtrière Charles Foix - Division Montyon, 47-83 Bd de l'Hôpital, 75651 Paris Cedex 13, France. E-mail: valery.trosini-desert@psl.aphp.fr

Support Statement: The "Association Pour la Vie-Espoir Contre le Cancer" provided financial support in the acquisition of bronchial endoscopic ultrasound equipment by the Groupe Hospitalier Pitié-Salpêtrière Charles Foix Dept of Respiratory and Critical Care Medicine (Paris, France).
Acknowledgements: The authors thank D. Khayat (Assistance Publique - Hôpitaux de Paris, Groupe Hospitalier PitiéSalpêtrière Charles Foix, Dept of Medical Oncology, Paris, France) and the "Association Pour la Vie - Espoir Contre le Cancer" (Paris) for their assistance in the acquisition of bronchial endoscopic ultrasound equipment by the Groupe Hospitalier Pitié-Salpêtrière Charles Foix, Dept of Respiratory and Critical Care Medicine.

\section{REFERENCES}

1 Ophir J, Cespedes I, Ponnekanti H, et al. Elastography: a quantitative method for imaging the elasticity of biological tissues. Ultrason Imaging 1991; 13: 111-134.

2 Barr RG, Destounis S, Lackey LB 2nd, et al. Evaluation of breast lesions using sonographic elasticity imaging: a multicenter trial. J Ultrasound Med 2012; 31: 281-287.

3 Shuzhen C. Comparison analysis between conventional ultrasonography and ultrasound elastography of thyroid nodules. Eur J Radiol 2012; 81: 1806-1811.

4 Brock M, von Bodman C, Palisaar RJ, et al. The impact of real-time elastography guiding a systematic prostate biopsy to improve cancer detection rate: a prospective study of 353 patients. J Urol 2012; 187: 2039-2043.

5 Baldewsing RA, Schaar JA, Mastik F, et al. Local elasticity imaging of vulnerable atherosclerotic coronary plaques. Adv Cardiol 2007; 44: 35-61.

6 Cardoso AC, Carvalho-Filho RJ, Stern C, et al. Direct comparison of diagnostic performance of transient elastography in patients with chronic hepatitis B and chronic hepatitis C. Liver Int 2012; 32: 612-621.

7 Iglesias-Garcia J, Larino-Noia J, Abdulkader I, et al. Quantitative endoscopic ultrasound elastography: an accurate method for the differentiation of solid pancreatic masses. Gastroenterology 2010; 139: $1172-1180$

8 Puli SR, Reddy JB, Bechtold ML, et al. Accuracy of endoscopic ultrasound to diagnose nodal invasion by rectal cancers: a metaanalysis and systematic review. Ann Surg Oncol 2009; 16: 1255-1265.

$9 \mathrm{Xu} \mathrm{W}$, Shi J, Zeng X, et al. EUS elastography for the differentiation of benign and malignant lymph nodes: a meta-analysis. Gastrointest Endosc 2011; 74: 1001-1009.

10 Giovannini M, Botelberge T, Bories E, et al. Endoscopic ultrasound elastography for evaluation of lymph nodes and pancreatic masses: a multicenter study. World J Gastroenterol 2009; 15: 1587-1593.

11 Bauwens O, Dusart M, Pierard P, et al. Endobronchial ultrasound and value of PET for prediction of pathological results of mediastinal hot spots in lung cancer patients. Lung Cancer 2008; 61: 356-361.

\title{
Fludarabine in paediatric steroid-refractory inflammatory lung injury after stem cell transplantation
}

\section{To the Editor:}

In children, the incidence of pulmonary complications after haematopoietic stem cell transplantation (HSCT) varies from
$10 \%$ to $20 \%$. Noninfectious causes, such as idiopathic pneumonia syndrome (IPS), bronchiolitis obliterans syndrome (BOS) and bronchiolitis obliterans organising pneumonia (BOOP), are considered nonspecific inflammatory injuries to the lung [1, 2]. 
These may represent a form of alloimmune injury to the lung after HSCT.

The current view is that there is a three-step process in the development of alloreactivity: 1) tissue damage results in 2) a release of inflammatory cytokines, which results in 3) activation and influx of T-cells [3].

First-line treatments for nonspecific inflammatory lung injuries are intravenous corticosteroids and supportive care. In four steroid-refractory, nonspecific inflammatory lung injury patients, we studied the efficacy and toxicity of fludarabine given on clinical grounds as a second-line treatment, despite the presence of respiratory viral infections.

In our study, nonspecific inflammatory lung injury was defined as IPS, BOS and BOOP (also referred to as cryptogenic organising pneumonia) diagnosed by clinical symptoms, typical highresolution computed tomography changes and/or results of pulmonary function tests (PFTs), if feasible. Clinical symptoms were defined as cough, dyspnoea, fever and oxygen requirement. Three preschool study patients were not able to perform PFTs.

In patients with upper respiratory tract infection symptoms, nasopharyngeal aspirate (NPA) samples were obtained, testing for respiratory virus infection by PCR. Bronchoalveolar lavage (BAL) for bacterial/fungal cultures, viral PCR and galactomannan testing were performed as clinically indicated. Quantitative real-time PCR (TaqMan $\mathbb{R}$; Life Technologies Europe BV, Bleiswijk, the Netherlands) for Epstein-Barr virus, cytomegalovirus, human herpesvirus 6 and adenovirus, and galactomannan monitoring in plasma were performed twice a week.

All patients diagnosed with a nonspecific inflammatory lung injury were treated with a methylprednisolone pulse $\left(10 \mathrm{mg} \cdot \mathrm{kg}^{-1} \cdot \mathrm{day}^{-1}\right.$ i.v. for 3 days and $2 \mathrm{mg} \cdot \mathrm{kg}^{-1} \cdot$ day $^{-1}$ prednisone thereafter, tapering by $25 \%$ per week to $0.5 \mathrm{mg} \cdot \mathrm{kg}^{-1} \cdot \mathrm{day}^{-1}$ ). The methylprednisolone pulses were repeated every 4 weeks until a positive clinical response was observed, up to a maximum of six courses. Other immunosuppressive agents were continued and adjusted to standardised levels. Along with immunosuppressive therapy, supportive care was provided. Patients were considered refractory to steroids when no positive clinical response, or even progressive lung injury, was seen.

A positive clinical response was defined as resolved pneumonia symptoms (dyspnoea, cough and fever) without additional oxygen requirement and/or, if feasible, improvement/normalisation of PFTs compared with the baseline values acquired at the start of lung injury. An absolute increment of $>12 \%$ in FEV1 from baseline can be regarded as significant and an increment of $8-12 \%$ can be regarded as borderline [4]. Computed tomography radiology was used for diagnosing nonspecific inflammatory lung injury but not to document response while clinical symptoms improved because of the additional radiation and anaesthesia burden. A partial response was defined as a sustained improvement in either clinical symptoms and/or oxygen supply but without a return to baseline values.

Between 2008 and 2010, in four patients with steroid-refractory, nonspecific inflammatory lung injury, methylprednisolone pulses were replaced by fludarabine as the immunosuppressive treatment. Corticosteroids were tapered while fludarabine was given at a dose of $30 \mathrm{mg} \cdot \mathrm{m}^{-2}$ every 3 weeks, up to a maximum of six courses. In addition, possible adverse events (e.g. cytopenia, alopecia and nausea) were recorded.

Initially, all four HSCT patients showed good donor cell engraftment and functioning of the graft. Patients developed the first signs of lung injury at a median of 2 months after transplantation (range 2-8 months) (table 1). In three patients, respiratory viruses were detected in BAL and/or NPAs before standard steroid therapy (threshold cycle $\left(C_{T}\right)$ values $<30$ for rhinovirus in two patients and adenovirus in one patient). One patient was positive for galactomannan in BAL and showed $\mathrm{BK}$ viraemia.

These patients with steroid-refractory, nonspecific inflammatory lung injury were treated with fludarabine, started at a median of 5 months after transplantation (range 3-10.5 months) (table 1). A positive clinical response was apparent in three patients after a median of four courses of fludarabine (range 2-4 courses). These patients showed a sustained response with neither clinical symptoms nor extra oxygen supply after a median follow up time of 21 months (range 5-36 months). The only adverse event noted was mild anaemia in patient 2 during the treatment courses, which resolved spontaneously 2 months after the last course of fludarabine. All patients showed distinct T-cell depletion with normalisation over time except for patient 4 who suffered from autoimmune cytopenia, for which he was treated with rituximab (table 1 and figure 1). Viral load (expressed in $\mathrm{C}_{\mathrm{T}}$ values) remained high in all patients but did not show an increase during treatment with fludarabine and no associated systemic viral disease was noted in these patients.

In patient 1 , the symptoms of tachy- and dyspnoea resolved with supplemental oxygen $\left(0.5 \mathrm{~L} \cdot \mathrm{min}^{-1}\right)$ during the night. She died of relapse of her disease $1.5 \mathrm{yrs}$ after the last fludarabine infusion without any sign of pulmonary symptoms at that time.

Patient 2 is alive and in complete remission to date, without any respiratory symptoms, while during her fludarabine courses, she suffered from coughing, and tachy- and dyspnoea, and needed supplemental oxygen supply during the day and night.

Patient 3, who has Hurler syndrome, was tachy- and dyspnoeic, and needed supplemental oxygen $\left(2 \mathrm{~L} \cdot \mathrm{min}^{-1}\right)$ when diagnosed with nonspecific inflammatory lung injury, despite steroid therapy. Fludarabine was started on the same day that he became respiratory insufficient, for which he needed mechanical ventilation. After 1 week, we decided to give fludarabine again because a clinical worsening associated with increasing T-cells was seen. Subsequently, after the second course of fludarabine, weaning from mechanic ventilation was possible and he was extubated after 2 weeks. Due to his pre-existing pulmonary condition, he still remains oxygen dependent during the day $\left(0.25 \mathrm{~L} \cdot \mathrm{min}^{-1}\right)$ and shows pulmonary symptoms for which he is ventilated overnight. Capillary blood gas analyses were indicative for chronic respiratory insufficiency with metabolic compensation (base excess $5-15 \mathrm{mmol} \cdot \mathrm{L}^{-1}$ ) of respiratory acidosis (carbon dioxide tension $50-65 \mathrm{mmHg}$ ). HRCT 3 months after fludarabine treatment showed increasing ground-glass opacities with air trapping, confirming the diagnosis bronchiolitis obliterans.

Patient 4 died from his lymphoma and combined immunodeficiency complicated by a nonpulmonary Aspergillus infection 17 months after transplantation and 5 months after the last 


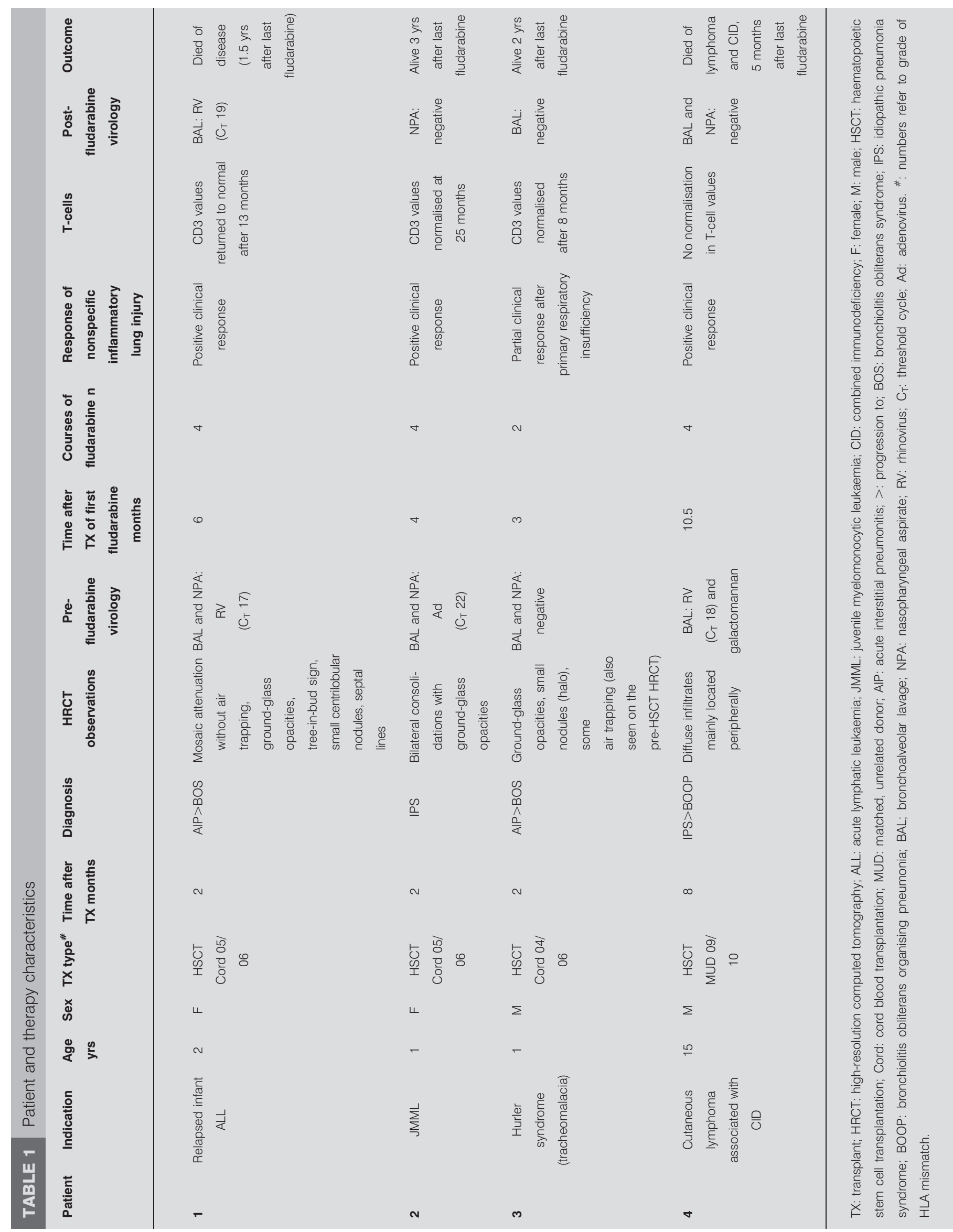



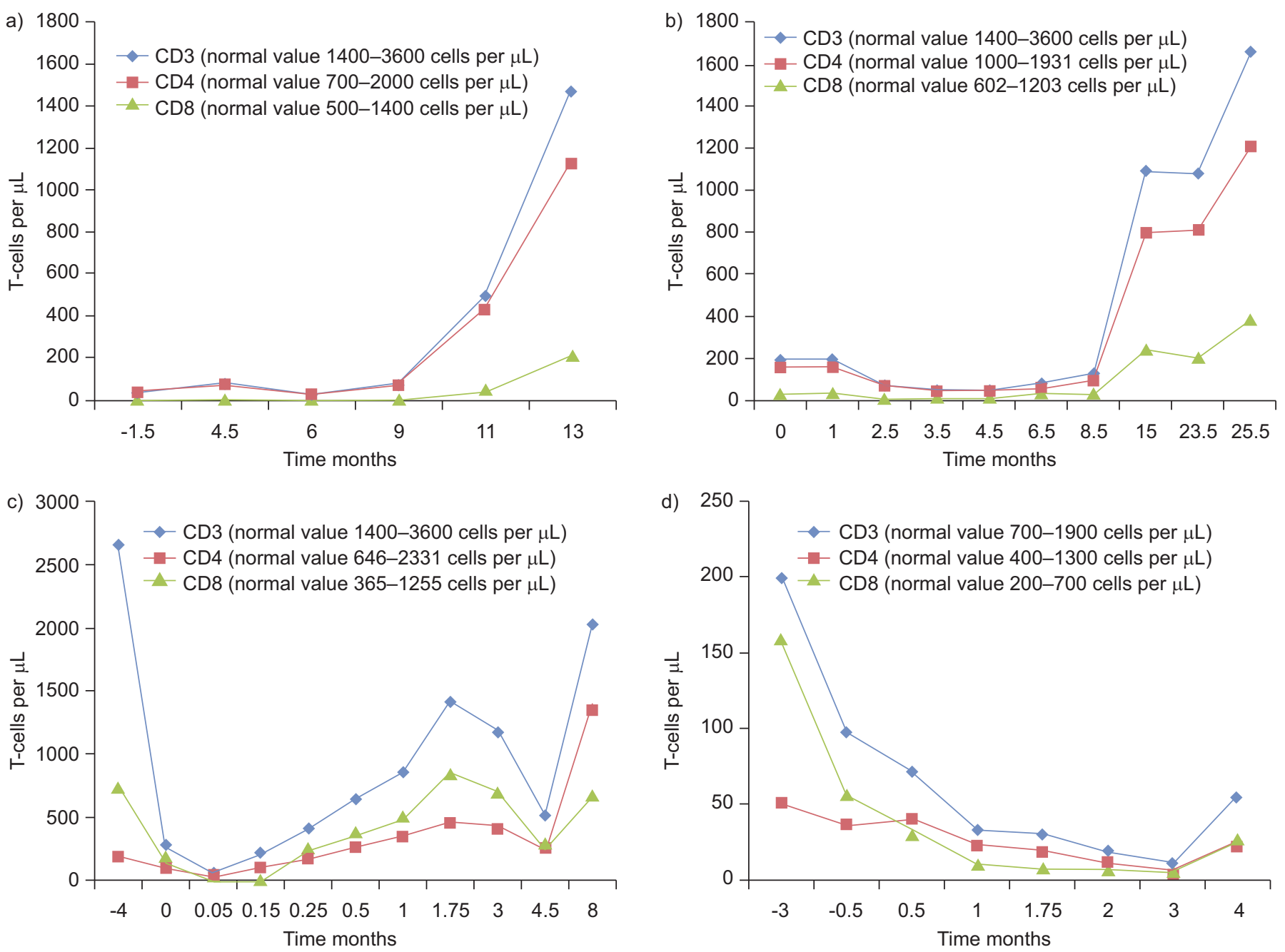

FIGURE 1. T-cell counts over time after the start of fludarabine $(t=0)$. a) Patient 1 ; b) patient 2 ; c) patient 3 ; d) patient 4

fludarabine course. At that time, he suffered from no pulmonary symptoms as seen during the fludarabine courses (tachypnoea, dyspnoea, cough and additional oxygen supply). PFTs showed an overall increase of $65 \%$ in FEV1 from $1.32 \mathrm{~L}$ (at time of lung injury diagnosis) to $2.19 \mathrm{~L}$ in the following 5 months.

Fludarabine as an immunosuppressive treatment modality was chosen as second-line therapy since this is suggested to be a relatively low-toxicity option leading to a deep T-cell depletion [5]. This impact on T-cells might dampen the alloreactivity in this nonspecific inflammatory lung injury. Increasing immunosuppression seems controversial when viral infections are present, though a higher exposure to immunosuppression (because of acute graft-versus-host disease) was associated with a lower susceptibility for alloimmune lung syndromes in HSCT recipients, despite the presence of respiratory viral infections [6].

Although the inflammatory process after lung transplantation (LTX) may be very different from that after HSCT, because other immune mechanisms besides T-cells are active [7], based on the success with fludarabine in patients after HSCT, fludarabine was given to a patient with steroid-refractory, biopsy-proven bronchiolitis obliterans 4.5 months after LTX for surfactant C deficiency. After Fludarabine treatment, the patient improved clinically, with less dyspnoea, a decrease in supplementary $100 \%$ oxygen from 2 to $1 \mathrm{~L} \cdot \mathrm{min}^{-1}$ by nasal cannula and a $24 \%$ increase in FEV1 from 0.21 to $0.26 \mathrm{~L}$ for 2 months. In addition, in this patient, there was a high load of respiratory viruses, which remained stable after three fludarabine courses. No side-effects, such as cytopenia, nausea or alopecia, were noted.

In summary, we demonstrated that $\mathrm{T}$-cell depletion with fludarabine led to a positive clinical response in three cases with steroid-refractory, nonspecific inflammatory lung injury after HSCT and a possible clinical response in another, despite the presence of respiratory viral infections and without significant side-effects.

Martine F. Raphael*, Bart L. Rottier" and Jaap Jan Boelens *Dept of Paediatric Hematology and Oncology, Wilhelmina Children's Hospital, University Medical Center Utrecht, "Paediatric Blood and Marrow Transplantation Program, Wilhelmina Children's Hospital, University Medical Center Utrecht, Utrecht, and "Dept of Paediatric Pulmonology and Allergology, Beatrix Children's Hospital, University Medical Center Groningen, Groningen, the Netherlands. 
Correspondence: M.F. Raphael, Dept of Paediatric Hematology and Oncology, Wilhelmina Children's Hospital, University Medical Center Utrecht, Lundlaan 6, 3584 EA Utrecht, the Netherlands. E-mail: m.f.raphael-2@umcutrecht.nl

Statement of Interest: None declared.

\section{REFERENCES}

1 Peters SG, Afessa B. Acute lung injury after hematopoietic stem cell transplantation. Clin Chest Med 2005; 26: 561-569.

2 Afessa B, Peters SG. Chronic lung disease after hematopoietic stem cell transplantation. Clin Chest Med 2005; 26: 571-586.

3 Reddy P. Pathophysiology of acute graft-versus-host disease. Hematol Oncol 2003; 21: 149-161.
4 Pellegrino R, Viegi G, Brusasco V, et al. Interpretative strategies for lung function tests. Eur Respir J 2005; 26: 948-968.

5 Gassner FJ, Weiss L, Geisberger R, et al. Fludarabine modulates composition and function of the T cell pool in patients with chronic lymphocytic leukemia. Cancer Immuno Immunther 2011; 60: 75-85.

6 Versluys $\mathrm{AB}$, Rossen JW, van Ewijk B, et al. Strong association between respiratory viral infection early after hematopoietic stem cell transplantation and the development of life-threatening acute and chronic alloimmune syndromes. Biol Blood Marrow Transplant 2010; 16: 782-791.

7 Saini DJ, Weber S, Ramachandran D, et al. Alloimmunity-induced autoimmunity as a potential mechanism in the pathogenesis of chronic rejection of human lung allografts. J Heart Lung Transplant 2011; 30: 624-631.

\section{Isolation of cells from the lower airways in infants with wheeze by sputum induction}

\section{To the Editor:}

Preschool wheezing affects up to $50 \%$ of children [1, 2], of whom approximately half will have stopped experiencing wheezy episodes by the age of 6 yrs [2]. Results from large cohort studies [2, 3] raise several important questions. First, when facing a child with preschool wheeze, how can we predict the natural history and identify those children who are going to grow out of their asthma by mid-childhood and distinguish them from those who develop classical, adult-type asthma? Secondly, how can we identify those children who are likely to benefit from treatment with inhaled corticosteroids, leukotriene receptor antagonists or both, in the absence of reliable immunological criteria underpinning these decisions? Airway inflammation is a key feature of asthma and is detectable in preschool children [4]. Obtaining lower-airway samples from preschool children usually involves bronchoalveolar lavage sampling under general anaesthesia and is therefore reserved for children with problematic severe asthma or where the diagnosis is in doubt [4].

An alternative method of obtaining samples noninvasively from the lower airways is by sputum induction [5]. The use of induced sputum in infants has been limited [6-8] and inflammatory cell profiles have not been reported. MuSSAFFI et al. [6] performed the procedure in young children with cystic fibrosis and reported that the procedure was well tolerated, and there was a high success rate in nonexpectorating patients. Successful use of sputum induction in the diagnosis of tuberculosis was reported in 142 out of 149 infants with a median age of 9 months [7]. The aim of our pilot study was to investigate the feasibility of obtaining lower airway samples adequate for quantification of cell types from young infants with a history of wheezing, by sputum induction.

Infants aged 0-2 yrs requiring one or more hospital admissions with wheezing or wheezing episodes resulting in referral to a specialist paediatric respiratory clinic were recruited. Infants with other significant medical conditions were excluded. Written parental consent was obtained. The local research ethics committee (Leicestershire, Northamptonshire and Rutland REC 1, Nottingham, UK; reference number 06/Q2501/18) approved all aspects of this study.

Families attended our infant lung function laboratory once the child had been free from respiratory tract infection or wheeze for $\geqslant 2$ weeks. Infants were examined by a paediatrician (A. Whittaker) and, if they were well and baseline arterial oxygen saturations were $\geqslant 95 \%$ in room air, the infant was studied. They were allowed to fall asleep naturally; however, four very active children were given a mild sedative (chloral hydrate, $50 \mathrm{mg} \cdot \mathrm{kg}^{-1}$ body weight, by mouth). When settled, the infants were given inhaled salbutamol (200 $\mu$ g given via metered-dose inhaler and spacer) 10 min prior to sputum induction with $4 \%$ nebulised hypertonic saline, delivered through a high-flow nebuliser and facemask for $2 \mathrm{~min}$. A sputum sample was collected by suctioning via a soft, small-bore catheter inserted through the nasopharynx.

The sputum was processed within $2 \mathrm{~h}$ of collection at $4{ }^{\circ} \mathrm{C}$ as described previously [9]. Cytospins were prepared using $75 \mu \mathrm{L}$ cell suspension at $40 \times g(450 \mathrm{rpm})$ for 6 min using a Shandon III cytocentrifuge (Shandon Southern Instruments, Sewickley, PA, USA). Two air-dried slides were stained using the DiffQuik method (Dade Behring, Düdingen, Switzerland) for calculation of the leukocyte differential cell count. Initially, the percentage of squamous cells in the sample was estimated by counting 300 successive cells from a representative section of the slide. Following this, a differential leukocyte count was established by counting 300 nonsquamous cells. Cell counts were performed by an investigator (A. Tellebati) who was blinded to the clinical patient data. A detailed review was undertaken (E.A. Gaillard and J. Grigg) under different levels of magnification. The slides were also reviewed by a paediatric 